\title{
Fluorescence Dot Counting Efficiency in Radiance Mapped Images
}

\author{
S. Shah* and F. A. Merchant** \\ *Quantitative Imaging Laboratory, University of Houston, Department of Computer Science, \\ Houston, TX 77204-3010 \\ **ADIR, LLC., 2450 South Shore Blvd., Suite 305, League City, TX 77573.
}

Chromosomal aberrations are a variation from the normal, either in structure or number of chromosomes, which result from an exchange of genetic material between two or more chromosomes or from a rearrangement of genetic sequences contained in a single chromosome. The analysis of such aberrations can be useful both in a clinical and in a toxicological context. A particularly useful cytogenetic technique for the analysis of aberrations is Fluorescence in situ Hybridization (FISH) [1]. FISH relies on the use of fluorescent dyes that are attached to the DNA probes and when excited by one wavelength of light, emit light at a second, longer, wavelength. FISH signals in interphase cells become visible as colored dots. In general, the emission spectra of most fluorophores are broad and partially overlapping. In typical FISH study, three fluorescent dyes are used, one to stain the cell nuclei, and two to detect DNA sequences. Although FISH is widely used for genetic analyses, its reliability and accuracy depends on the types of probes, cells and their fixation. The major disadvantage associated with the use of fluorescent dyes is the weak signal emanating from a cell or its labeled constituents. In typical studies, a background pixel consists of 5000 photons, a DAPI-stained nucleus pixel is an additional 3000 photons, and a labeled chromosome pixel can be an additional 10000 photons. These numbers are at least a factor of 100 smaller than the values that are obtained for images acquired through a conventional CCD camera at room-level illumination [2]. Several investigators have evaluated the hybridization efficiencies of interphase FISH using probes specific for chromosomes 13, 18, 21, X and Y. The results reported vary from $60 \%$ to $98 \%$ for $\mathrm{XY}$ detection and $42 \%$ to $88 \%$ for autosomal aneuploidy detection depending on the type of cells and the choice of probes [3]. These results can be acceptable for rapid detection of chromosome aneuploidies, however, when the cells sought represent a very low proportion of the total cell population, a very high efficiency is desired while maximizing specificity and sensitivity.

In this paper, we present an approach to improve the efficiency of detecting fluorescent signals in FISH images by recovering the radiance map of the CCD camera. In general, there exists a nonlinear relationship between the true radiance of the fluorescent probe and the detected pixel value. We use a simple technique to recover the response curve by using a set of images taken with varying but known exposure durations [4]. This allows us to generate an image with increased dynamic range that covers the full extent of the radiance captured by all the images. We further analyze the distribution of each of the fluorescent signals and remap the image values to generate a high contrast image with compressed dynamic range. This leads to images where saturated values are attenuated while the weak signals are amplified. We used 20 interphase FISH slides labeled with DAPI, FITC, and Texas Red to validate the efficiency of our approach. Using a single view on a slide, a series of images at exposures varying were 0.03 seconds to 1.4 seconds in intervals of 0.04 seconds were collected. These images formed the input for recovering the camera response curve. If $E_{i}$ is the irradiance value at pixel $i, \Delta t_{j}$ is the set of known exposures, $Z_{i j}$ is the image pixel value at spatial index $i$ at exposure $j$, the camera response function $f$ can be computed as $Z_{i j}=f\left(E_{i} \Delta t_{j}\right)$. 
Assuming $f$ to be monotonic, the irradiance $E_{i}$ and the inverse function of $f$ can be solved in the least-square error sense for by rewriting the above equation as $g\left(Z_{i j}\right)=\ln E_{i}+\ln \Delta t_{j}$ where $g=\ln f^{-1}$. Once the response curve $g$ is recovered, it can be used to quickly convert pixel values to relative radiance values, assuming the exposure $\Delta t_{j}$ is known. We compute the curve based on one set of images and use the same to recover the radiance values for every other image taken while imaging all the cells. A representative set of images taken to recover the response curve and the corresponding response curves for the three colors are shown in figure 1. Image on the left in figure 2 shows the original image while the image on the right has been remapped. The remapped image presents higher signal to noise to improved identification of fluorescent dots. By testing our approach on 2000 FISH images, we found that the dot counting specificity and sensitivity can be improved from $76 \%$ to $94 \%$.

\section{References}

[1] D. Pinkel et al., Cytogenetic analysis using quantitative high sensitivity fluorescence hybridization. Proc. Nat. Acad. Science, 83 (1986) 2934-2938.

[2] H. Netten et al., FISH and Chips: Automation of Fluorescence Dot Counting in Interphase Cell Nuclei. Cytometry, 28 (1997) 1-10.

[3] P.N. Rao et al., Rapid detection of aneuploidyin in uncultured chorionic villus cells using fluorescence in situ hybridization. Prenat. Diag., 13 (1993) 233-238.

[4] P.E. Debevec and J. Malik, Recovering high dynamic range radiance maps from photographs. SIGGRAPH, (1997).
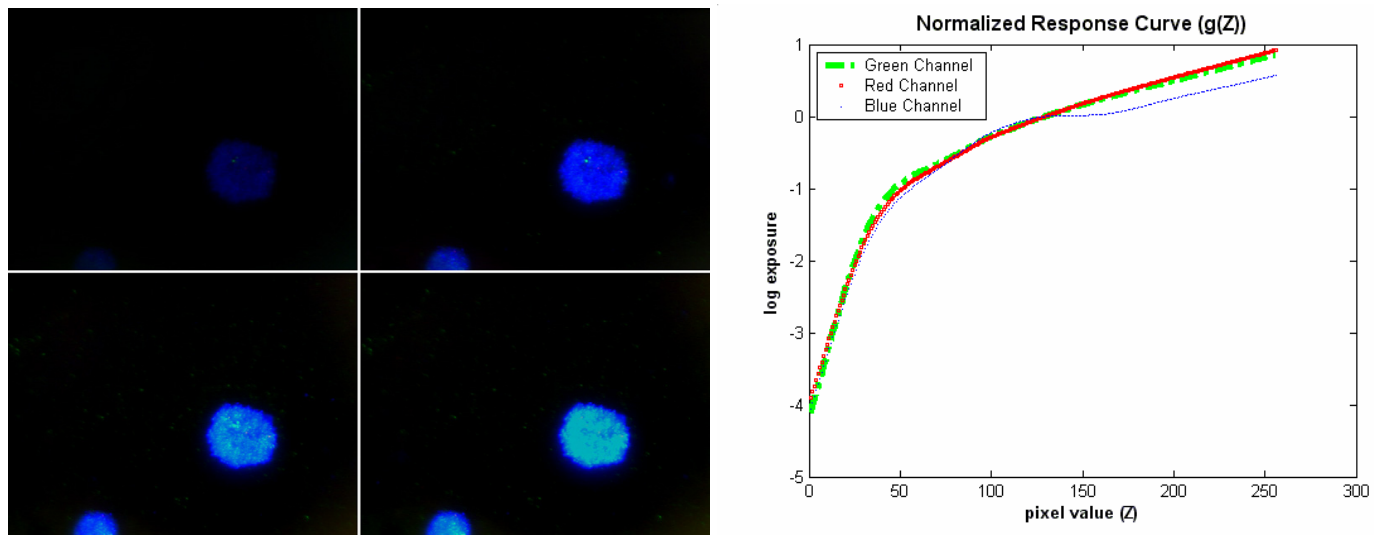

FIG. 1. Images acquired to recover the camera response curve at $0.1,0.5,1.0$, and 1.4 seconds (left) and the recovered camera response curve for the three colors (right).
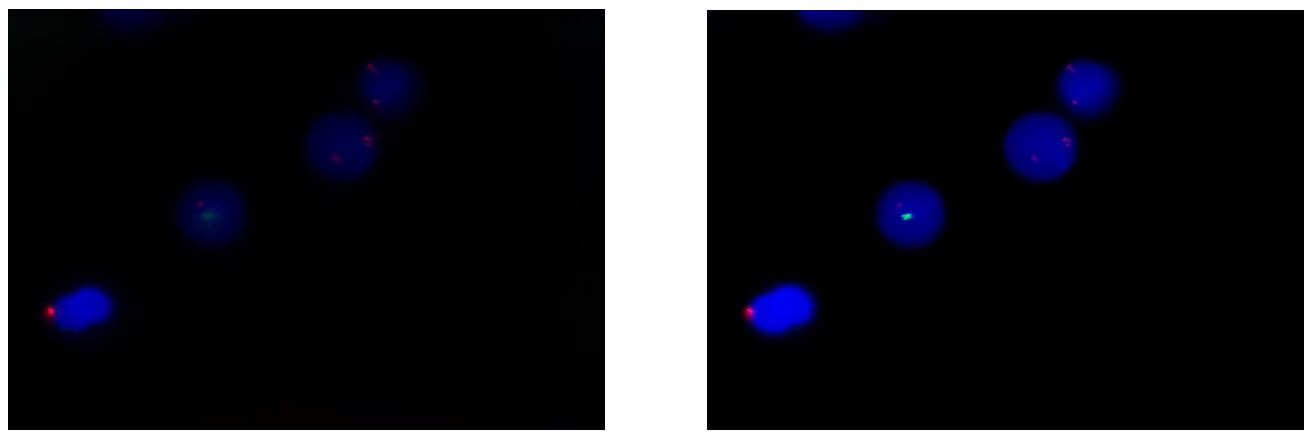

FIG. 2. Image on left is the original data captured at 0.5 seconds and the image on the right is the radiance mapped data exhibiting an increased signal-to-noise ratio across all three fluorophores. 